\title{
Trinken, bevor der Durst kommt?
}

\section{Flüssigkeitsbedarf von Sportlern und kritische Betrachtung des Getränkeangebots}

\author{
Drinking Before you're Thirsty? \\ Athletes' Need for Fluids and Critical Review of Available Beverages
}

\section{Autor}

Institut

\section{H. Heseker}

Institut für Ernährung, Konsum und Gesundheit, Universität Paderborn

\section{Schlüsselwörter \\ - Wasserregulation \\ - Wassermangel \\ - Schweißverluste \\ - Sportgetränke \\ - isoton \\ - hypoton \\ hyperton \\ Keywords \\ - waterregulation \\ water deficiency \\ - sweat losses \\ - sports drinks \\ - isotonic \\ - hypotonic \\ - hypertonic}

\section{Bibliografie}

DOI http://dx.doi.org/

10.1055/s-0042-102730

Aktuel Ernahrungsmed 2016;

41, Supplement 1: S22-S26

(c) Georg Thieme Verlag KC

Stuttgart · New York

ISSN 1862-0736

\section{Korrespondenzadresse}

Prof. Dr. Helmut Heseker

Institut für Ernährung,

Konsum und Gesundheit,

Universität Paderborn

Warburger Straße 100

33098 Paderborn

Tel.: 05251/60-3835

helmut.heseker@upb.de

\section{Zusammenfassung}

$\nabla$

Das Durstempfinden des Menschen ist ein verlässlicher Sinn, der effektiv vor Dehydratation schützt. Trinken über den Durst hinaus ist physiologisch nicht notwendig. Um den Grundbedarf zu decken, sollten Erwachsene pro Tag 2-2,51 Wasser aufnehmen, davon stammen rund $1,5 \mathrm{l}$ aus Getränken. Der Zusatzbedarf und damit die optimale Trinkmenge richten sich nach der Schweißproduktion. Intensive sportliche Aktivität kann einen Wasserverlust von 4-101 bewirken, der ersetzt werden muss. Im Leistungssport ist darüber hinaus der Ersatz von Kohlenhydraten und Elektrolyten erforderlich. Trinkempfehlungen für Sportler zielen immer auf eine ausgeglichene Wasserbilanz. Gute Sportgetränke sorgen für eine schnelle Verfügbarkeit von Wasser, indem sie rasch aus dem Magen entleert und im Dünndarm absorbiert werden. Darüber hinaus liefern sie schnell verfügbare Zucker und Natrium. Isotone oder leicht hypotone Getränke mit 30-80 g Kohlenhydraten und 400-1100 mg Natrium pro Liter erfüllen diese Kriterien am besten. Hypertone Getränke wie Fruchtsäfte, Energydrinks oder Limonaden eignen sich nicht als Sportgetränke, da sie die Dehydratation kurzfristig verstärken. Auch natriumarmes Wasser führt zu einer Nettosekretion von Elektrolyten in das Darmlumen. Für die Anreicherung von Sportgetränken mit Vitaminen oder potenziell leistungsfördernden Substraten gibt es keine wissenschaftliche Basis.

Wasser ist der elementarste Nährstoff und muss täglich in ausreichender Menge zugeführt werden. Der Mensch kann je nach akkumulierter Energie Wochen und sogar Monate ohne feste Nahrung auskommen, überlebt aber nur wenige Tage ohne Wasser.

Wasser übernimmt im Organismus viele physiologische Funktionen. Es dient als Baustoff und

\section{Abstract \\ $\nabla$}

Human beings' thirst sensation is a reliable sense that protects us from dehydration in an effective manner. It is physiologically not necessary to drink more than one's thirst would indicate. In order to satisfy their basic fluid requirement, adults should drink 2-2.5 litres of water per day; of this amount, 1.5 litres comes from beverages. Any additional need and thus the optimal fluid intake depend on the production of sweat. Intensive exercise activity can result in a water loss of 4 10 litres, which will have to be replaced. Additionally, in competitive sports, carbohydrates and electrolytes need replacing. Recommendations for fluid intake for athletes always aim to ensure a good water-balance. Quality sports drinks ensure quick availability of water as they pass through the stomach quickly and are absorbed in the small bowel. Furthermore they provide quick available sugars and sodium. Isotonic or slightly hypotonic beverages containing 30-80g of carbohydrates and $440-1100 \mathrm{mg}$ of sodium per litre meet these criteria best. Hypertonic beverages, such as fruitjuices, energy-drinks, or lemonade are not suitable as sports-drinks because they increase dehydration in the short term. Water that is low in sodium also results in a net secretion of electrolytes into the bowel. No scientific basis exists for the fortification of sports-drinks with vitamins or potentially performance-enhancing substrates.

Kühlmittel, als Wärmeleiter und universelles Lösungsmittel. Darüber hinaus ermöglicht Wasser den Transport unzähliger Substanzen und ist Reaktionspartner für biochemische Reaktionen. Die fettfreie Körpermasse hat mit 73\% einen relativ konstanten Wassergehalt, während das Fettgewebe weniger Wasser enthält. Der höhere Körperfettanteil bei Frauen erklärt den im Vergleich zu 
Männern geringeren Wassergehalt: Frauen bestehen zu 45 - 55\%, Männer zu 50-60\% aus Wasser, Neugeborene zu 80\% [1, 2].

\section{Auf das Durstgefühl ist Verlass}

$\nabla$

Wasser ist im Körper unterschiedlich verteilt. Zwei Drittel sind intrazellulär gebunden, ein Drittel liegt im extrazellulären Raum. Da die Wasserversorgung essenziell ist, besitzt der Körper mehrere Regulationsmechanismen: Die Steuerung der Wasserausscheidung und damit die Feinregulation des Wasserhaushalts erfolgt durch hormonelle Signale an die Nieren. Konkret handelt es sich um das Renin-Angiotensin-Aldosteron-System (RAAS), das Antidiuretische Hormon (ADH) und Natriuretische Peptide (ANP). Außerdem gibt es zentralnervöse Mechanismen, die Durst auslösen. Dieses System ist insgesamt sehr verlässlich. Es springt bereits bei den ersten Anzeichen einer Dehydrierung an und löst Durst aus [3].

Bei Wassermangel steigt der osmotische Druck und führt über eine Aktivierung von Osmorezeptoren im Gehirn zur Freisetzung des Antidiuretischen Hormons (ADH, Vasopressin) in der Hypophyse. ADH aktiviert die Wasserrückresorption in der Niere, das fehlende Volumen wird wieder hergestellt und der Blutdruck steigt. Reichliche Flüssigkeitszufuhr und Alkoholgenuss hemmen die ADH-Freisetzung. Dies erklärt, dass exzessiver Biergenuss diuretisch wirkt [2].

\section{Wie viel Wasser braucht der Mensch? \\ $\nabla$}

Der tägliche Grundbedarf an Wasser beträgt 1/30 des Körpergewichts oder, anders ausgedrückt, $30-35 \mathrm{ml}$ pro Kilo Körpergewicht beziehungsweise $1 \mathrm{ml}$ pro kcal. Bei einem gesunden, $75 \mathrm{~kg}$ schweren Erwachsenen entspricht dies einer Menge von rund 2,51 pro Tag. Die tägliche Flüssigkeitsbilanz muss ausgeglichen sein. Von den zugeführten 2-2,51 Wasser pro Tag stammen rund 1,5l aus Getränken, 0,7 l aus der Nahrung und 0,3 1 aus dem Oxidationswasser, sprich aus der Verbrennung von Kohlenhydraten und Fetten. Rund 1,51 Wasser werden über den Urin ausgeschieden, dazu kommen 0,31 über die Atmung, 0,61 über die Haut und 0,11 über den Stuhl ( $\bullet$ Abb. 1) [4].

Es gibt Situationen, in denen der Wasserbedarf deutlich gesteigert ist. Dazu gehört körperliche Anstrengung in feucht-heißem Klima, in trockener, kalter Luft oder in großer Höhe. Mehr Wasser braucht der Mensch auch bei einem hohen Energieumsatz und starkem Schwitzen, bei erhöhter Körpertemperatur und Fieber. Auch Fastende haben einen erhöhten Wasserbedarf, weil das Oxidationswasser aus der Verbrennung fester Lebensmittel fehlt. Eine hohe Zufuhr an Kochsalz steigert den Wasserbedarf ebenfalls, genau wie gewisse pathologische Zustände, etwa Diarrhö und Erbrechen [2].

Der Zusatzbedarf an Wasser über den Grundbedarf hinaus richtet sich nach der Schweißproduktion: Auf einen Liter verdunsteter kommt etwa ein halber Liter abgetropfter Schweiß. Nur 25\% der aus Nährstoffen freigesetzten Energie kann für Muskelkontraktionen genutzt werden (mechanische Effizienz), 75\% der mobilisierten chemischen Energie wird in Wärme umgewandelt (thermische Effizienz). Diese Wärme muss abgeleitet werden, damit die Körperkerntemperatur nicht auf kritische Werte steigt. Bei einer Körperkerntemperatur von über $40^{\circ} \mathrm{C}$ werden Enzymaktivitäten beeinflusst, was sich schnell auf die Leistungsfähigkeit auswirkt.

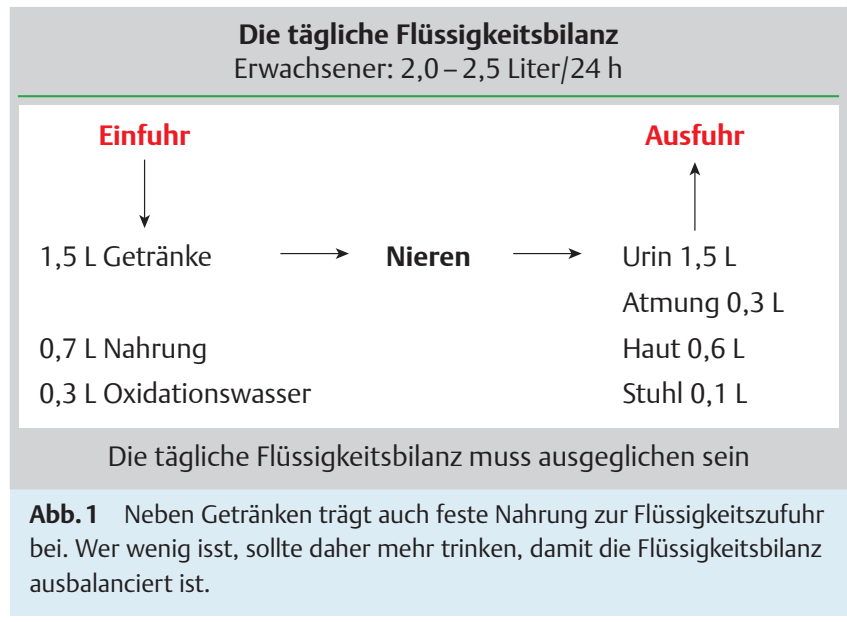

Schweißverluste werden in erster Linie durch die klimatischen Bedingungen beeinflusst, wie Hitze, Sonneneinstrahlung, hohe Luftfeuchtigkeit oder Saunabesuche. Weitere Einflussfaktoren sind die Intensität und Dauer einer körperlichen Belastung, ebenso wie der Trainingszustand: Gut Trainierte schwitzen deutlich weniger als Untrainierte. Beispielsweise sind gute Marathonläufer zwar oft dehydriert, spüren aber keine gesundheitlichen Einschränkungen, während untrainierte Marathonläufer aufgrund höherer Schweißverluste häufiger kollabieren. Die Veranlagung und das Geschlecht spielen ebenfalls eine Rolle: Männer schwitzen stärker als Frauen, da sie mehr Schweißdrüsen haben. Auch Aufenthalte in großer Höhe erhöhen die Schweißverluste. Dort ist der Sauerstoffpartialdruck niedriger, was zu einer schnelleren Atmung und infolgedessen zu einer vermehrten pulmonalen Perspiration führt. In kalter Umgebung kommt es dagegen zu einer peripheren Vasokonstriktion und Zentralisierung des Kreislaufs. In der Folge arbeiten die Nieren verstärkt und es kommt zur Diurese.

Bei intensiven sportlichen Belastungen können pro Tag durchaus 4-10l Wasser verloren gehen, dazu kommen Natriumverluste von 3,5-7g, die ausgeglichen werden müssen [5].

\section{Wassermangel und die Folgen}

Bei einem Wassermangel werden harnpflichtige Substanzen wie Salze und Harnstoff unvollständig ausgeschieden. Sinkt das Körpergewicht um 3\%, das entspricht etwa 21 Wasser, sind Schwäche und Störungen der Gehirnfunktionen mögliche Folgen. Bereits nach 2-4 Tagen ist die Austrocknung und Eindickung des Blutes so ausgeprägt, dass der Kreislauf zusammenbrechen kann [2].

Im Detail laufen dabei folgende Vorgänge im Körper ab: Das Gesamtvolumen des Blutplasmas sinkt, daraufhin verschlechtern sich die Fließeigenschaften des Blutes, das zentrale Blutvolumen und das Schlagvolumen des Herzens werden reduziert. Die Herzfrequenz steigt, das Herzminutenvolumen sinkt, ebenso die Hautdurchblutung und die Schweißbildung. In der Konsequenz steigt die Körperkerntemperatur ( $\bullet$ Abb.2).

Wassermangel macht sich sehr früh mit Symptomen bemerkbar. Bereits bei einem Wasserverlust von 1-3\% des Körpergewichts meldet sich Durst. Dies entspricht einem Defizit von etwa einem halben Liter. Gleichzeitig sinkt die Speichelsekretion, was sich als Mundtrockenheit äußert, und zudem geht die Harnproduktion zurück - ein Symptom, das mit bloßem Auge erkennbar ist: 


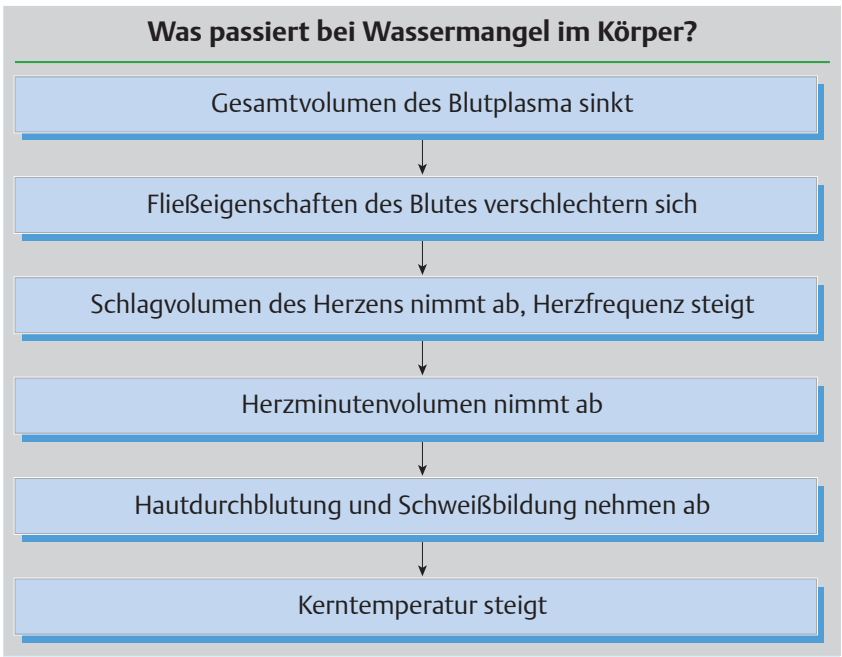

Abb.2 Dunkler Urin und ein trockener Mund sind sichere Zeichen für Wassermangel.

Dunkler Urin ist immer ein Zeichen für Wassermangel. Beträgt der Wasserverlust 4-6\% des Körpergewichts, kommt es zu Müdigkeit und Übelkeit, die Herzfrequenz steigt, ebenso die Körpertemperatur. Bei einem Verlust von 7-10\% treten Schwindel und Atemnot ein, das Blutvolumen sinkt. Bei 10\% Verlust kommt es zu Verwirrtheit, Krämpfen und Delirium, bei 20\% stirbt der Mensch [5].

Im Sport können je nach Sportart und -dauer, Umgebungstemperatur und Trainingszustand über Schweiß und Atemluft erhebliche Flüssigkeitsverluste auftreten, bis zu mehreren Litern pro Belastung. Bereits eine geringe Dehydratation mindert die Temperaturregulation und die Leistungsfähigkeit.

Nicht nur zu wenig, auch zu viel Wasser kann dem Körper zu schaffen machen. Gesunde Menschen haben zwar eine sehr hohe Toleranz; die Nieren sind in der Lage, pro Stunde fast 11 Wasser auszuscheiden. Dennoch kann eine akute Wasserintoxikation auftreten, wenn die maximale Ausscheidungsrate der Nieren durch schnelles Trinken großer Wassermengen überschritten wird. Das gilt vor allem für hypotone Getränke. In diesem Fall steigt die Extra- und Intrazellularflüssigkeit mit Hyponatriämie, die Zellen nehmen vermehrt Wasser auf und das Risiko für Muskelkrämpfe steigt [2].

\section{Ausgleich der Flüssigkeitsbilanz}

Der Ausgleich der Flüssigkeitsbilanz ist essenziell und hängt hauptsächlich von der Magenentleerungsrate und der Wasserabsorption ab. Beide Faktoren werden durch die Zusammensetzung des Getränks beeinflusst [5, 6].

Die Wasserabsorption erfolgt größtenteils im Dünndarm und ist an die Absorption von Glukose und Natrium gekoppelt. Die Absorption hängt von der Magenentleerungsrate $a b$. Je schneller das Wasser vom Magen in den Darm gelangt, desto besser. Einfluss auf die Magenentleerungsrate haben folgende Faktoren:

- Konzentration und Molekulargewicht der enthaltenen Teilchen (Osmolalität).

- Glukosekonzentration: Bei Werten über 5\% ist die Entleerungsrate verzögert, die Flüssigkeit bleibt länger im Magen.
- Konzentration an Saccharose und Maltodextrinen: Die Entleerungsrate verzögert sich erst bei einer Konzentration von $7-10 \%$

- Getränketemperatur: Optimal für die Entleerungsrate sind $5-10^{\circ} \mathrm{C}$, besser verträglich sind allerdings $15-20^{\circ} \mathrm{C}$ warme Getränke.

- Flüssigkeitsvolumen: Je stärker der Magen gefüllt ist, desto schneller erfolgt die Magenentleerung.

- Organische Säuren, zum Beispiel in Fruchtsäften, verzögern die Magenentleerung.

\section{Gute Sportgetränke sind hypo- oder isoton}

Im Dünndarm wird Wasser absorbiert, strömt umgekehrt aber auch vom Blut ins Darmlumen. In welchem Umfang, hängt von der Konzentration der osmotisch aktiven Teilchen im Darmlumen ab. Die höchste Netto-Wasserabsorption ist bei leicht hypotonen bis isotonen Lösungen zu beobachten.

Diese Lösungen enthalten 3-7\% Glukose und 400mg Natrium pro Liter. Isotone Getränke haben eine ähnliche Osmolalität wie Blut (280-290 mosmol), hypotone Getränke eine ähnliche wie Schweiß (unter 270 mosmol). Hypertone Getränke enthalten dagegen über $330 \mathrm{mosmol}$ - und damit ist die Konzentration osmotisch aktiver Teilchen so hoch, dass die Sekretionsrate von Wasser höher ist als die Absorptionsrate. Das heißt, es strömt mehr Wasser aus dem Blut ins Darmlumen als umgekehrt [7, 8].

Fazit: Zuckerhaltige, hypo- oder isotone Getränke werden am schnellsten absorbiert. Die maximale Wasserabsorption liegt bei 0,9 Liter pro Stunde. Bei hohen Schweißverlusten findet unter Umständen während der Belastung kein vollständiger Ersatz statt. Beispielsweise kann die Körperkerntemperatur bei untrainierten Marathonläufern auf $40^{\circ} \mathrm{C}$ steigen und die Leistung mindern.

Hypertone Getränke werden langsamer aus dem Magen entleert, langsamer absorbiert und oft weniger gut vertragen. Sie verstärken vorübergehend die Dehydratation. Das bedeutet, reine Fruchtsäfte oder klassische Erfrischungsgetränke mit 10-12\% Zucker sind ungeeignet im Sport. Auch natriumarmes Wasser führt zu einer Nettosekretion von Elektrolyten in das Darminnere.

\section{Trinkempfehlungen für Sportler \\ $\nabla$}

Trinkempfehlungen für Sportler zielen auf eine ausgeglichene Wasserbilanz. Galt in den 1970er- und 80er-Jahren die Empfehlung, vor dem Sport so viel wie möglich zu trinken, ist dies heute obsolet. Vor dem Training oder Wettkampf setzt man auf Prähydratation, das heißt, Sportler sollten 4 Stunden vor dem Training, Spiel oder Wettkampf 5-7 ml natriumhaltige Flüssigkeit pro Kilo Körpergewicht langsam trinken. Ist der Urin 2 Stunden vor der Aktivität noch stark konzentriert, können sie weitere 3$5 \mathrm{ml}$ pro Kilo Körpergewicht trinken $[6,8]$.

Während der Aktivität ist die Rehydratation wichtig: Bei intensiver Belastung können Sportler ad libitum $400-800 \mathrm{ml}$ pro Stunde trinken, wobei $800 \mathrm{ml}$ nur bei ruhigen Körperbewegungen wie Radrennen möglich sind. Für Sportarten mit Erschütterungen, beispielsweise Laufen, gilt die Empfehlung, 15-20 min vor der Aktivität $100-150 \mathrm{ml}$ zu trinken ( $\bullet$ Abb.3).

Die optimale Trinkmenge muss jeder Sportler für sich herausfinden, einüben und einen an seine Bedürfnisse angepassten Trink- 


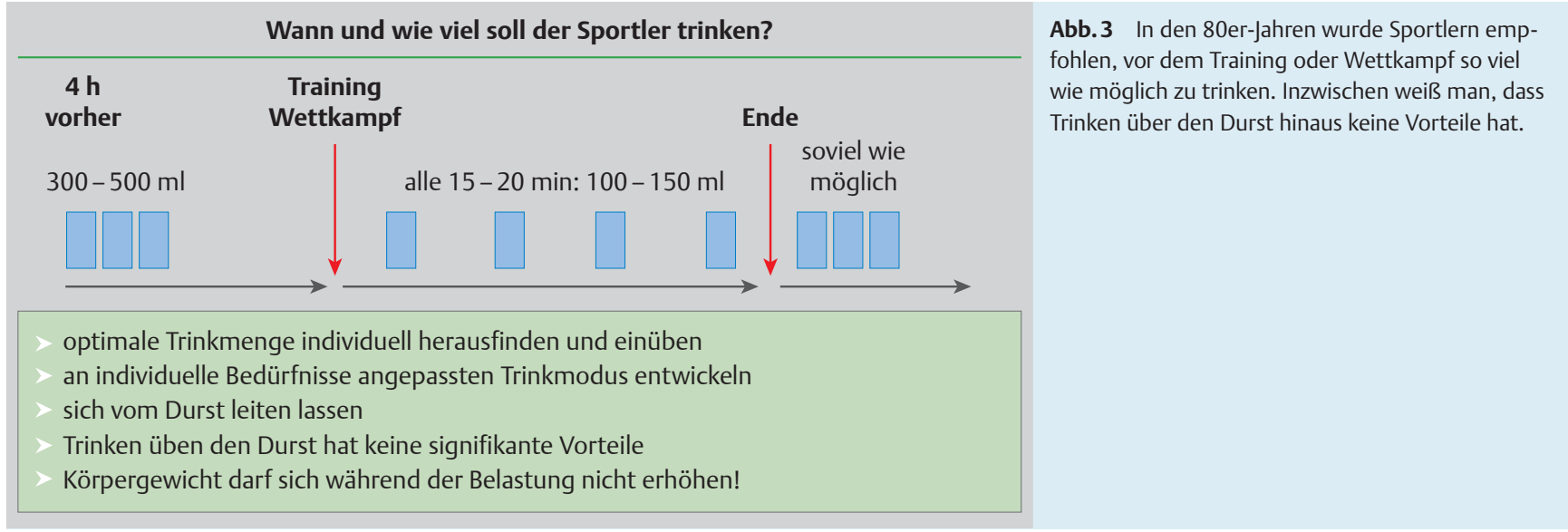

modus entwickeln. Dabei gilt die Empfehlung, sich vom Durst leiten zu lassen. Anders als lange vermutet, hat Trinken über den Durst hinaus keine signifikanten Vorteile [9]. Im Gegenteil sollte das Körpergewicht während der Belastung nicht steigen. Die Überkompensierung von Flüssigkeitsverlusten bedeutet zusätzliches Gewicht und lässt die Athleten im Wettkampf zurückfallen. Beim Marathon beispielsweise sind die Sieger nach dem Wettkampf oft deutlich stärker dehydriert als die Verlierer [10]. In der Regenerationsphase nach dem Sport hängt die Trinkempfehlung vom Grad der Dehydratation ab. Ist das Körpergewicht um weniger als $5 \%$ reduziert und steht in den folgenden 24 Stunden keine erneute Belastung an, können Sportler nach Belieben Flüssigkeit und Elektrolyte ersetzen.

Bei starker Dehydratation nach einer Ausdauerbelastung von über $90 \mathrm{~min}$ und bei geplanter erneuter Belastung ist es sinnvoll, mit Beginn der Erholungsphase sofort so viel isotonische Getränke aufzunehmen, bis die Gewichtsabnahme kompensiert ist.

\section{Was macht ein gutes Sportgetränk aus?}

$\nabla$

Gute Sportgetränke sollen für eine schnelle Verfügbarmachung von Wasser sorgen, werden rasch aus dem Magen entleert und im Dünndarm absorbiert. Zweitens liefern sie Kohlenhydrate, um eine Ermüdung hinauszuzögern und drittens Natrium zum Ausgleich der Verluste im Schweiß. Diese Kriterien erfüllen isotone oder leicht hypotone Getränke am besten, die dem Athleten schmecken und gut verträglich sind. Sie enthalten 30-80 g Kohlenhydrate pro Liter, am besten als Gemisch aus Glukose und Fruktose. Der Natriumgehalt liegt zwischen 400 und $1100 \mathrm{mg}$ pro Liter, wobei ab $800 \mathrm{mg}$ ein salziger Geschmack dominiert und zu Akzeptanzproblemen führt [7, 8].

Viele Sportgetränke enthalten hoch dosierte Vitaminzusätze, etwa Vitamin C. Diese sind unnötig und möglicherweise sogar nachteilig für die schnelle Regeneration. Abzulehnen sind außerdem Zusätze von potenziell leistungsfördernden Substraten; es gibt keine wissenschaftliche Basis, die deren Einsatz rechtfertigen würde. Ebenfalls ungeeignet sind stark koffeinhaltige Energydrinks, reine Fruchtsäfte, Limonaden und Malzbier. Ihr Kohlenhydratgehalt ist zu hoch, sie sind stark hyperton und bewirken eine kurzfristige Dehydratation mit Leistungseinbruch. Alkoholfreie Weizenbiere sind zwar isoton, enthalten aber statt Natrium viel Kalium - und damit den falschen Mineralstoff.

Die europäische Behörde für Lebensmittelsicherheit (EFSA) hat inzwischen für Sportgetränke beantragte Health Claims bewer- tet. Anerkannt wurden Claims zur Verbesserung der Wasserabsorption während des Sports sowie zur Beibehaltung von Ausdauerleistungen, jeweils für die Zielgruppe „aktive Personen beim Ausdauersport“. Als Begründung führte die EFSA an, dass die Urasche-Wirkungs-Beziehung zwischen dem Konsum von Kohlenhydrat-Elektrolyt-Lösungen und der verbesserten Wasserabsorption bzw. der Beibehaltung von Ausdauerleistungen während des Sports belegt sei.

Abgelehnt dagegen wurde der Claim „Reduzierung von gefühlter Kraftanstrengung während des Sports“ für die Zielgruppe „aktive Personen, die Sport treiben“ [11].

\section{Fruchtsaftschorle als ideales isotones Sportgetränk} $\nabla$

Auf dem Markt erhältliche Sportgetränke sind meist isoton oder leicht hypoton; die Osmolalität liegt im Bereich zwischen 240 und $320 \mathrm{mmol} / \mathrm{kg}$. Im Vergleich dazu hat Orangensaft eine Osmolalität von 600, Apfelsaft von 730 und Cola von $500 \mathrm{mmol} / \mathrm{kg}$. Diese hypertonen Getränke sind nicht geeignet für Sportler. Werden Fruchtsäfte 1:1 mit Wasser verdünnt, sinkt die Osmolalität auf isotone Werte - und werden damit zu geeigneten Sportgetränken.

Generell gilt: Leistungssportler, die an ihre Grenzen gehen, profitieren von Sportgetränken, weil sie im intensiven Training oder Wettkampf die Leistungsfähigkeit verbessern. Die breite Masse der Freizeitsportler dagegen braucht sie nicht, im Gegenteil: Wer joggt, um abzunehmen, sollte auf die Kohlenhydratkalorien in Sportlergetränken verzichten und besser nur Wasser trinken. Eine nach individuellen Bedürfnissen zusammengestellte Saftschorle ist für moderate Belastungen vollkommen ausreichend.

\section{Interessenkonflikt}

$\nabla$

Der Autor hat keinen Interessenkonflikt.

\section{Literatur}

1 Stahl A, Heseker H. Wasser. Ernährungs-Umschau 2006; 53: 353 - 357

2 Baily JL, Sands JM, Franch HA. Water, Electrolytes, and Acid-Base Metabolism. In: Ross AC et al., Hrsg. Modern Nutrition in Health and Disease. Philadelphia: Lippincott Williams \& Wilkins; 2014: 102 -132

3 Rehner G, Daniel H. Biochemie der Ernährung. 3. Auflage. Heidelberg: Spektrum Akademischer Verlag; 2010

4 Deutsche Gesellschaft für Ernährung (DGE), Österreichische Gesellschaft für Ernährung (ÖGE), Schweizer Gesellschaft für Ernährungsfor- 
schung (SGE), Schweizerische Vereinigung für Ernährung (SVE). Referenzwerte für die Nährstoffzufuhr. 2. Auflage, 1. Ausgabe. Neustadt: Neuer Umschau Buchverlag; 2015

5 American College of Sports Medicine. ACSM Position Stand. Exercise and fluid replacement. Med Sci Sports Exerc 2007; 39: 377-390

6 Heseker $H$. Über den Sinn und Unsinn von Sportgetränken. Ernährungs-Umschau 2013; 60: M40-M43

7 ETH Zürich Swiss Forum for Sport Nutrition. Dehydratation. http:// www.sfsn.ethz.ch/sportnutr/tipps/Drinking/Zuwenig (Zugriff am 26.11.2012)
8 IOC. Nutrition for athletes. A practical guide to eating for health and performance. 2003: http://www.olympic.org/Documents/Reports/EN/ en_report_833.pdf (Zugriff am 26.11.2012)

9 Noakes TD. Is drinking to thirst optimum? Ann Nutr Metabol 2010; 57 (Suppl. 02): 9-17

10 Trautwein S, Hartwich M, Schulze Uphoff $U$ et al. Hirnödem durch Marathonlauf. Notfall und Rettungsmedizin 2009; 12: $287-289$

11 EFSA. Scientific opinion on the substantiation of health claims related to carbohydrate-electrolyte solutions. EFSA Journal 2011; 9: 2211 